\title{
Successful Multimodality Management of Refractory Cancer Pain on Shoulder Caused by Lung Cancer Metastasis and Resistant to Analgesics: A Case Report
}

\author{
Liya $\mathrm{Hu}^{1}$, Qian $\mathrm{Chu}^{2}$, Yuan Chen², Peng Zhang ${ }^{2}$, Lei Zhou ${ }^{2}$, and Suqiong Ji ${ }^{3 *}$ \\ ${ }^{1}$ Department of Geriatrics, Huazhong University of Science and Technology, China \\ ${ }^{2}$ Department of Oncology, Hua Zhong University of Science and Technology, China \\ ${ }^{3}$ Department of Neurology, Hua Zhong University of Science and Technology, China
}

*Corresponding author: Suqiong Ji, Department of Neurology, Tongji Hospital, Tongji Medical College, Hua Zhong University of Science and Technology, Jiefang Avenue 1095, Wuhan, 430030, China.

Received Date: February 18, 2020

Published Date: March 05, 2020

\begin{abstract}
s
Background: This case describes a successful management of patient suffered from refractory cancer pain and with poor response to any analgesic.

Case Presentation: A 61-year-old woman suffered from severe refractory shoulder pain cause by lung cancer metastasis with bone destruction, pancoast syndrome and local soft tissue invasion. Opioid and other medication is completely ineffective, accompanied with obvious side effect of severe drowsiness.

Case Management: Multiple department treatment (MDT) group were initiate to evaluate the main component of pain and etiological therapy: 1) bone metastases pain; 2) Neuropathic Pain cased by brachial plexus invasion; 3) compression pain associated with soft tissue swelling. Palliative bone radiotherapy, neurosurgical intervention and sodium aescin were successively used due to the patient's changing symptoms.

Case Outcome: The patient was successfully relived from the pain remission sustained for 5 month. NRS score reduced from 8 to 2 with low dose of opioid and no side effect of drowsiness.

Conclusion: Successful and personalized management of refractory cancer pain depends on the evaluation of changing symptoms, etiology analysis and multidisciplinary teamwork. New approaches are recommended to pain management, especially the axillary nerve pulse radiofrequency surgery.
\end{abstract}

Keywords: Refractory cancer pain; Pancoast syndrome; Lung cancer; Metastasis; Neuropathic pain; Pain management

\section{Introduction}

Cancer pain is an inevitable symptom burden for $66 \%$ of cancer patients [1]. The traditional cancer pain management is depending on the principle of three-step analgesic ladder developed by WHO in 1986, which recommends the administration of opioid and nonopioid analgesic [2]. It has been the corner stone for cancer pain control. Even though, the World Health Organization showed that a significant minority of patients (10\%-15\%) still do not attain adequate pain relief. For those refractory cancer pain, even with much higher dose of opioid, it may sometimes still couldn't relieve. And high dose of opoid analgesic often accompanied with obvious drowsiness, constipation and dizzy. It altogether contributes to deterioration of quality of life. Cancer pain is never a homogenous term, it includes a large variety of pain conditions, characterized by different etiology and pathological mechanisms. Particularly, for terminal phase of cancer patients, pain is often difficult to control due to its mixed nature. Individualized pain management has been emphasized over the years. Multidisciplinary assessment and 
treatment are one of the important means. Here we report a case of successful multimodality management of refractory shoulder pain caused by severe lung cancer metastasis, and resistant to analgesics.

\section{Case Presentation}

A 61-year-old female patient was diagnosed with adenocarcinoma of the right lung (60\% solid and $40 \%$ acinar) stage II (T2 N0 M0) 2 years before, confirmed through surgery. EGFR 19/21 WT, ALK (-). After 4 cycles of adjuvant chemotherapy (Pemetrexed $800 \mathrm{mg}$ and cisplatinum $120 \mathrm{mg}$ ), She entered the follow-up period. 1 year later, She called she had suffered from aggravating shoulder pain, presented with persistent and severe right shoulder pain, along with slight paraesthesia and numbness in the right arm. And she couldn't go to sleep for the whole week (NRS 8). After admission to hospital, multiple bone and liver metastasis were found out by bone ECT and MRI scan. The cancer relapse. She was diagnosed of terminal cancer of stage IV lung cancer with bone and liver metastasis.

Of note, her shoulder pain were so serious. Since the beginning, she has sustained pain in the right scapula, accompanied by intermittent paroxysmal electric shock-like pain. She was unable to lie flat because of the severe pain in the back shoulder, and suffered difficulties of raising the right upper limb, with slight swelling and numbness. But there is no concave edema, no skin temperature elevation, no cyanosis, pale and other skin color changes. NRS scores 8.

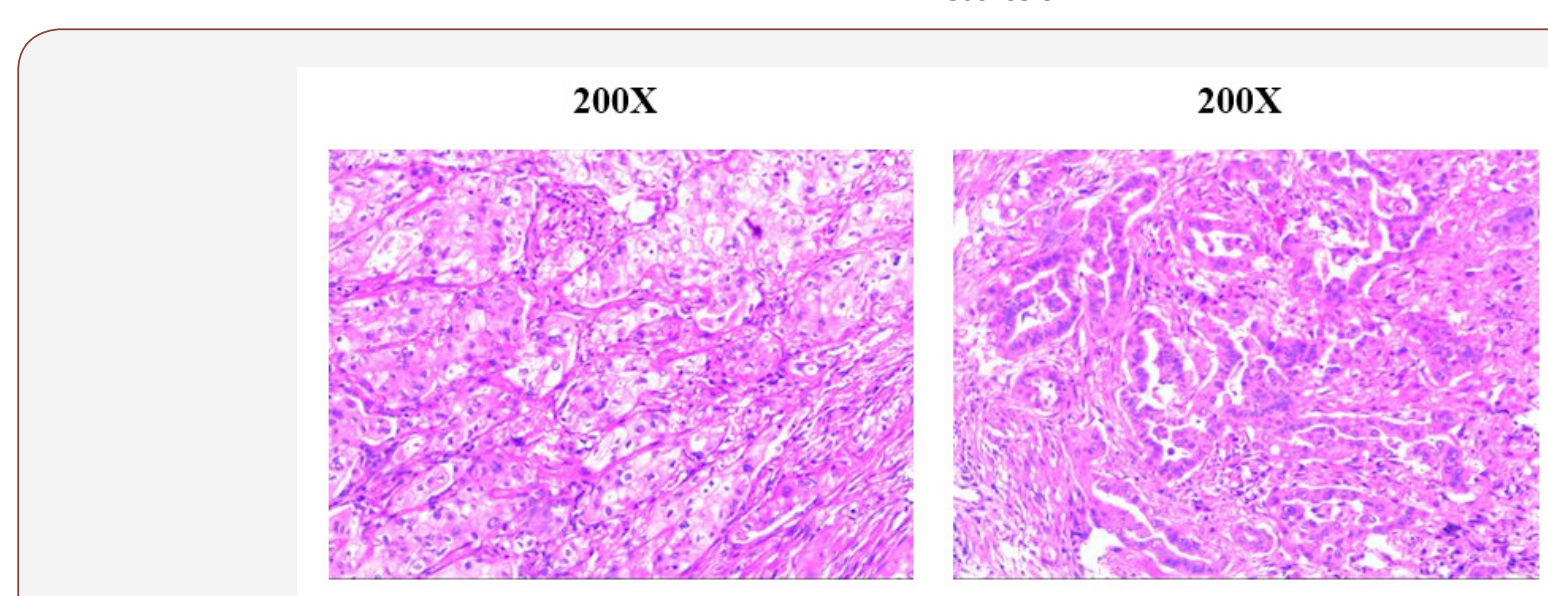

Figure 1 Representative pathologic images of the main components of the patient's tumor by hematoxylin and eosin (HE) staining. (A) solid component of lung adenocarcinoma; (B) acinar component of lung adenocarcinoma. 20X, 20 times amplified.

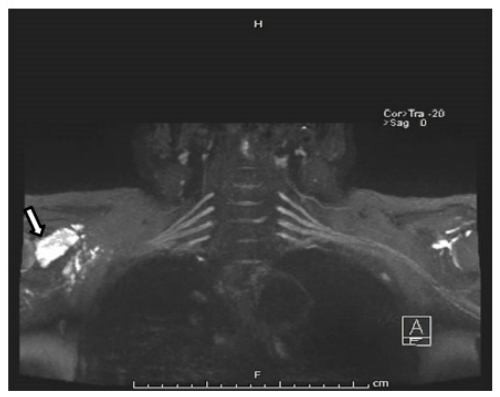

A



B

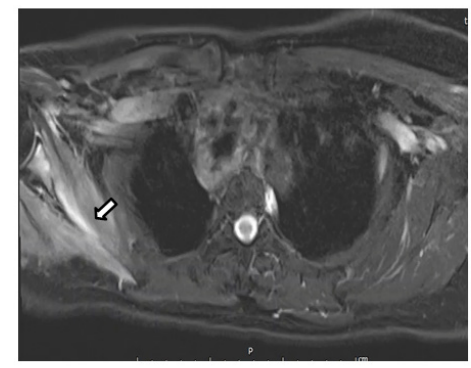

C

Figure 2 Representative MRI for the patient. Arrows indicates: (A) bone metastasis of scapula; (B) brachial plexus invasion; (C) soft tissue swelling in scapular region.

Citation: Liya Hu, Qian Chu, Yuan Chen, Peng Zhang, Lei Zhou, et al. Successful Multimodality Management of Refractory Cancer Pain on Shoulder Caused by Lung Cancer Metastasis and Resistant to Analgesics: A Case Report. Arch Neurol \& Neurosci. 7(3): 2020. ANN. 


\section{Investigations}

After admission to hospital, CT scan showed no solid mass on lung and mediastinum. Multiple bone destruction was found identically on the right scapula, together with aggressive mass, vague margin and locally soft tissue tumefaction. Bone ECT scan further confirmed scapula bone and another non-weight-bearing metastasis.

Figure 3 Timeline of the clinical course and related pain symptom scored by NRS. Patient presented poor response to opioid. At the beginning, the NRS remained 8-9 with opioid 100mg Q8h along with NSAIDs (Celecoxib) 100mg q12h. The traditional palliative radiotherapy of spealbone didn't relieve much of the pain. After nerve symptoms and tissue edema appeared overtime, the neurosurgical intervention and sodium aescin were consecutively conducted, and the NRS score was significantly decreased to 1-2 and the pain relieved to a great extent till now.

\section{Treatment}

6 cycles of DP (Docetaxol+Avastin, Q3W) plus Avastin was given from 2018.10.21 to 2019.03.26, after that she continued with Docetaxol+Avastin maintenance treatment (Q3W). The disease was assessed as SD till now. Since the pain were so severe, the pain team was early involved in the management of our patient care, focusing on providing relief from this severe shoulder pain.

Firstly, regularly palliative radiotherapy of bone targeted to scapula is given. There was no significant remission after local radiotherapy (300cGy*10F) of the right shoulder, we continue to add another 300cGy*2F. During this period, the dose of opioid (OxyContin) were 100mg Q8h along with NSAIDs (Celecoxib) $100 \mathrm{mg}$ q12h. However, after the whole radiotherapy (total 3600cGy*12F), her pain has just a little relieved. Meanwhile, the side effect of opioid of drowsiness were so obvious that she fell asleep in the bathroom for one time during opioid treatment. Her compliance of opioid use is getting worse and worse, as well as the pain control. The NRS scores 6-7.

We doubted that the pain was not just caused by the bone destruction. At the same time, we noticed that the patient complained more and more frequently about the electric shock-like pain on her right arm. After following the advice from neurology department, the patient was convinced to get an MRI of brachial plexus. MRI showed the bone destruction. However, apart from this, the surrounding muscle was edema, and the axillary segment of the right brachial plexus was compressed. After consulting with the neurology department, they suppose the compression of the brachial plexus nerve is now the main component of the pain. Based on this, we utilized Ultrasound guided axillary nerve pulse radiofrequency surgery by neurology department and anesthesia department. After that, the patient reported that the pain and the shock-like pain from right arm was relieved significantly. The NRS score were reduced from 8 to 3 or 4 . The dose of opioid was decreased to $60 \mathrm{mg} \mathrm{q} 12 \mathrm{~h}$ along with NSAIDs.

However, there was still swelling on the shoulder from the MRI, even though there is no visible swelling from the surface of the skin. In addition, she reported that the degree of swelling was closely related to pain. Tension-like swelling on shoulder is usually paroxysmal accompanied by a significant increase in pain. Therefore, we added sodium aescin to eliminate edema, hoping to relieve compression caused by edema and further relieve pain. Finally, the pain was further alleviated. The dose of opioids was reduced to $30 \mathrm{mg} \mathrm{Q} 12 \mathrm{~h}$, and drowsiness side effects completely disappeared. The NRS score were reduced to 1-2.

\section{Discussion}

In most cases, it is very difficult to relieve intractable pain just through opioids and nonopioid analgesics. In this case, we encountered a patient suffered from refractory cancer with poor response to opioids. We conducted radiotherapy, surgical intervention and anti-edema drug to relieve the patient's sever shoulder pain step by step through MDT, depending on the patient's changing symptoms and etiology of pain. There appears to be three main types of pain of our patient over time: 1) bone metastases pain; 2) Neuropathic Pain cased by brachial plexus metastasis; 3 ) compression pain associated with soft tissue swelling. We provide a special and successful case regarding dynamic and continuous multi dimension management of refractory cancer pain. 
According to a recent review, it could be summarized to four main types of pain classified by etiology, that is neoplastic damage to bone and joints, damage to viscera, damage to soft tissue and miscellaneous and to lesions of nervous tissue [3]. Occasionally, pain could be complex and mixed. In a study of 464 hematological cancer patients, 284 different pain syndromes were reported, of which $8 \%$ were mixed [4]. The identification of the pain category through etiology, could help us to predict prognosis and guide therapeutic interventions. Refractory cancer pain usually comes in form of mixed pain, especially mixed with neuropathic component. However, to identify neuropathic component is not so easy because mixed pain is usually characteristically different from classical neuropathic pain. In clinical practice, patient-reported outcomes are helpful in identifying patients with possible neuropathic pain. Pain could be evaluated by questionnaires, such as the McGill Pain Questionnaire and the Brief Pain Inventory, which have been shown to be sensitive to pain change in large trials. Several other scales are also recommended specifically for neuropathic pain, such as the Leeds Assessment of Neuropathic pain, Pain ID, the Neuropathic Pain Symptom Inventory, the Neuropathic Trial Symptom Scale, and the Neuropathic Pain Scale. While most of the screening tools for neuropathic pain is established for patients with chronic non-cancer pain. There is still no standard clinical assessment of neuropathic pain among cancer patients. The Leeds Assessment of Neuropathic Symptoms and Signs (LANSS), and painDETECT has been validated among cancer patients in some studies. While Takahiro doubted that both screening tools for neuropathic pain (Pain DETECT and S-LANSS) could not identify the neuropathic component of mixed pain among patients with tumor-related cancer pain, especially when pain was moderate or severe [5]. Applicable assessment tools were still urgently needed especially for mixed pain involving neuropathic component.

Neuropathic pain (NP) is present in about one third of patients with cancer pain [6]; Cancer pain driven by neuropathic mechanism is resistant or at least less responsive to opioids [7]. Pancaost syndrome is one of the common etiology of NP among cancer patients. MR imaging is a useful technique choice for the assessment of brachial plexopathies. Current practice started to suggest interventional therapies used along with standard analgesic regimes to improve analgesia or reduce adverse effects [8]. Interventional therapies include radiotherapy, dorsal root rhizotomy, cordotomy of the spinothalamic tract, sympathectomy, thalamotomy, myelotomy, nerve ablation, regional local anesthetic infusion, loose technique, acupuncture and other techniques. Ultrasound guided axillary nerve pulse radiofrequency surgery is an emerging neurosurgical intervention for cancer pain treatment in recent years. Compared with traditional treatment, the temperature produced by pulsed radiofrequency is less than 42 ${ }^{\circ} \mathrm{C}$, which selectively result in the activity loss on pain conduction fiber and will not effect the annatomy structure of nerve fibers. Thus it avoids the complications of decreased muscle movement and esthesiodermia. Raslan's study reported that $98 \%$ of patients had complete or partial satisfactory pain relief, with $80 \%$ of patients maintaining good results at 6-month follow-up. There were no complications related to motor weakness [9]. Nowadays, ultrasound-guidance has made nerve ablation a safe procedure.

Inflammatory edema and the infiltration of inflammatory mediators is also involved in the pathogenesis of pain. Sodium aescin are the rings triterpenoid saponins, which belongs to nonsteroidal anti-inflammatory drugs. Sodium aescinate has been widely used in clinical to detumescence, anti-inflammatory, and to improve blood circulation. From MRI, we can see the local swelling pressure is obvious. After treatment of sodium aescinate, hypertonia were decreased, and thus significantly ease the pain. We suggest sodium aescinate to be considered and listed as an auxiliary analgesic, while more compellent clinical data were still needed.

Pain related to cancer metastasis often raises complex management problem. Its complexity not only depends on the type of pain, but also on the biological characteristics of tumor. Adenocarcinoma is one of the main histologic type of lung cancer. It consist five major sub-types, lepidic, acinar, papillary, solid and micropapillary. To date, studies have reported unfavorable prognosis associated with solid predominant (SP) histological subtype [10]. Both DFS and OS of solid type tumors are reportedly worse than partial solid type tumors in the same clinical T category $[11,12]$. Considering these evidences, Kinoshita even suggested the 8th TNM classification could be further improved [13]. This suggested that lung adenocarcinoma with solid components is more aggressive. Pain caused by this type of tumor metastasis should be more complex and needs more attention, especially for those who don't have EGFR, ALK and other clinically significant mutations for targeted therapy and immunotherapy.

In conclusion, we report a case of sever refractory pain due to lung cancer metastasis of scapula, local muscle tissue and brachial plexus, which present in a cluster of dynamic symptoms over time. Depending on the changing symptoms and etiology analysis is the key to a more personalized pain management. New approaches are recommended including targeted multimodal analgesia, interventions to pain management and increasing use of the multidisciplinary team and support services.

\section{Acknowledgment}

We would like to thank Peng Zhang and Lei Zhou for the data collection of CT, MRI and biopsy imaging. Apart from those, we would like to thank Dr. Yuan Chen, Dr Qian Chu for English language editing and valuable suggestions on this manuscript.

\section{Author Contribution}

- Data collection: Suqiong Ji, Peng Zhang, Lei Zhou.

- Writing-original draft: Liya Hu and Suqiong Ji.

- Writing-review \& editing: Yuan Chen, Qian Chu, Li Zhang. 


\section{Conflict of Interest}

No conflict of interest.

\section{References}

1. Van den Beuken-van MH, Hochstenbach LM, Joosten EA, Tjan-Heijnen VC, Janssen DJ (2016) Update on Prevalence of Pain in Patients with Cancer: Systematic Review and Meta-Analysis. J Pain Symptom Manag 51: 1070-1090.

2. WHO Expert Committee on Cancer Pain Relief and Active Supportive Care \& World Health Organization (1990) Cancer pain relief and palliative care: report of a WHO expert committee [meeting held in Geneva from 3 to 10 July 1989]. World Health Organization.

3. Bouhassira D, Luporsi E, Krakowski I (2017) Prevalence and incidence of chronic pain with or without neuropathic characteristics in patients with cancer. Pain 158: 1118-1125.

4. Niscola P, Tendas A, Scaramucci L, Giovaninni M, Cupelli L, et al. (2011) Pain in malignant hematology. Expert Rev Hematol 4: 81-93.

5. Higashibata T, Tagami K, Miura T, Okizaki A, Watanabe YS, et al. (2019) Usefulness of pain DETECT and S-LANSS in identifying the neuropathic component of mixed pain among patients with tumor-related cancer pain. Support Care Cancer 28(1): 279-285.

6. Caraceni A, Shkodra M (2019) Cancer Pain Assessment and Classification. Cancers (Basel) 11(4)
7. Bechakra M, Moerdijk F, van Rosmalen J, Koch BCP, van der Rijt CCD, et al. (2018) Opioid responsiveness of nociceptive versus mixed pain in clinical cancer patients. Eur J Cancer 105: 79-87.

8. Pravardhan Birthi, Paul Sloan (2013) Interventional Treatment of Refractory Cancer Pain. The Cancer Journal 19(5): 390-6.

9. Harsh V, Viswanathan A (2013) Surgical/radiological interventions for cancer pain. Curr Pain Headache Rep 17: 331.

10. Jiang T, Li M, Lin M, Zhao M, Zhan C, et al. (2019) Meta-analysis of comparing part-solid and pure-solid tumors in patients with clinical stage IA non-small-cell lung cancer in the eighth edition TNM classification. Cancer Manag Res 11: 2951-2961.

11. Kinoshita F, Toyokawa G, Matsubara T, Kozuma Y, Haratake N, et al. (2019) Prognosis of Early-stage Part-solid and Pure-solid Lung Adenocarcinomas. Anticancer Res 39: 2665-2670.

12. Miyahara N, Nii K, Benazzo A, Hoda MA, Iwasaki A, et al. (2019) Solid predominant subtype in lung adenocarcinoma is related to poor prognosis after surgical resection: A systematic review and metaanalysis. Eur J Surg Oncol 45: 1156-1162.

13. Bennett MI, Rayment C, Hjermstad M, Aass N, Caraceni A, et al. (2012) Prevalence and aetiology of neuropathic pain in cancer patients: a systematic review. Pain 153: 359-365. 